Pacific Journal of Mathematics

THE BOUNDEDNESS OF THE CANTOR-BENDIXSON ORDER
OF SOME ANALYTIC SETS 


\section{THE BOUNDEDNESS OF THE CANTOR-BENDIXSON ORDER OF SOME ANALYTIC SETS}

\section{R. Daniel Mauldin}

Let $X$ and $Y$ be complete separable metric spaces (Polish spaces). If $E$ is a subset of $X \times Y$, and $x \in X$, then by the $x$-section of $E, E_{x}$, is meant $E \cap(\{x\} \times Y)$. By $P_{S}(E)$ is

$$
P_{S}(E)=\left\{x: E_{x} \text { is scattered }\right\} \text {. }
$$

In this paper the following uniform boundedness principle for the Cantor-Bendixson order of analytic sets will be demonstrated.

THEOREM L. Let $W$ be an analytic subset of $X \times Y$ and let $M$ be an analytic subset of $X$ such that $M \subset P_{S}(W)$. Then there is a countable ordinal $\alpha$ such that the $\alpha$ th CantorBendixson derived set of $E_{x}$ is empty, for each $x$ in $M$.

Let us recall that if $A$ is a subset of $X$, then the CantorBendixson derivatives of $A$ may be defined by transfinite induction as follows:

$$
A^{(0)}=A
$$

$A^{(\alpha)}=\bigcap_{\beta<\alpha}\left\{x: x\right.$ is an acculumation point of $A^{(\beta)}$ and $\left.x \in A\right\}$.

Recall that a subset, $H$, of a Polish space is scattered if, and only if $H$ is a countable $G_{\delta}$ set, or equivalently, there is a countable ordinal $\gamma$ such that the $\gamma$ th Cantor-Bendixson derived set, $H^{(r)}$, of $H$ is empty [5]. By the Cantor-Bendixson order of a subset $H$ of a topological space is meant the first ordinal $\gamma$ such that $H^{(\gamma)}=H^{(\gamma+1)}$. The Cantor-Bendixson order of every subset of a Polish space is necessarily less than $\omega_{1}[5]$.

If $E \subset X \times Y$ and $M \subset X$, then $E$ will be bounded on $M$ provided there is an ordinal $\gamma, \gamma<\omega_{1}$, such that for each $x$ in $M$, the CantorBendixson order of $E_{x}$ is $\leqq \gamma$; otherwise $E$ will be said to be unbounded on $M$.

Let us note that in order to prove Theorem $\mathrm{L}$ it suffices to show that if $E$ is an analytic subset of $X \times Y$ such that each $x$-section of $E$ is scattered then $E$ is bounded on the $X$ projection of $E$, $\pi_{X}(E)$.

Theorem $\mathrm{L}$ has the following corollary:

Corollary 1. Let $X$ be an uncountable Polish space. Let $\mathscr{C}$ be any class of countable $G_{o}$ subsets of $X$ which contains all the countable compact subsets of $X$ except possibly countably many. Then no analytic set in $X^{2}$ can be universal for $\mathscr{C}$. 
Corollary 1 is proven by A. S. Kechris and D. A. Martin in [3]. Also, Corollary 1 has as corollaries:

COROLLARY 2. There is no analytic set in the plane $R^{2}$ which is universal for the countable closed subsets of $R$.

COROLlary 3. There is no Borel set in $R^{2}$, which is universal for the countable $G_{\dot{o}}$ subsets of $R$.

Corollaries 2 and 3 are proven by D. G. Larman and C. A. Rogers in [6]. A proof of Corollary 2 is given in the first section of this paper which involves techniques which differ from those used in [3], [6] and in the following parts of this paper.

The techniques of proof used in the second and third sections of this paper follow those used by Lusin in his deep book [7]. In fact, on page 186 of [7], Lusin states Theorem $\mathrm{L}$ for the space $R^{n} \times R$. However, Lusin does not give a proof.

In the second section a proof of another theorem of Lusin's is given $[7$, p. 247]:

TheORem C. Let $A$ be an analytic subset of $X \times Y$ such that each $X$-section of $A$ is countable. Then $A \subset B$, where $B$ is a Borel subset of $X \times Y$ such that each $X$-section of $B$ is also countable.

The proof given here is in a slightly more general setting than Lusin's and the binary sieve of Lebesgue is used here instead of "the elementary sieve" as used by Lusin.

Theorem $\mathrm{C}$ is the major key to the proof given here of Theorem L.

Some definitions and notations are now given.

Let $E$ be a subset of $X \times Y$ and $M$ a subset of $X$.

If $\alpha$ is an ordinal, then

$$
D_{Y}^{(\alpha)}(E)=\bigcup_{x \in X}\left(E_{x}\right)^{(\alpha)} .
$$

A family $\mathscr{Z}$ of subsets of $X \times Y$ is said to be simultaneously unbounded with respect to $E$ on $M$ provided that for each $\gamma<\omega_{1}$, there is some $x \in M$ such that

$$
\left(H \cap D_{Y}^{(\gamma)}(E)\right)_{x} \neq \varnothing,
$$

for all $H \subset \mathscr{C}$.

If $0<\varepsilon_{1}<\varepsilon_{2}$ and $\Gamma \subset X \times Y$, then the $\varepsilon_{1}$-band of $\Gamma$ with respect to $E$ is: $B_{\varepsilon_{1}}(\Gamma ; E)=\{(x, z) \in \Gamma: \exists(x, y) \in E$ with the distance from $y$ to $z$ less than $\left.\varepsilon_{1}\right\}$; the $\left(\varepsilon_{1}, \varepsilon_{2}\right)$-ring of $\Gamma$ with respect to $E$ is; 
$R_{\varepsilon_{1}}^{\varepsilon_{2}}(\Gamma, E)\{(x, z) \in \Gamma: \exists(x, y) \in E$ such that the distance from $y$ to $z$ is between $\varepsilon_{1}$ and $\varepsilon_{2}$.

It is a pleasure to thank Robert R. Kallman for many valuable discussions concerning descriptive set theory.

Before proceeding to the arguments, the author would like to pose two of the problems which have occurred to him while working on this paper;

Is there an analytic subset of $I \times I$ which is universal for the family of all analytic subsets of $I$ of Lebesgue measure zero? of the first category?

\section{Applications of the Arsenin-Kunugui theorem.}

In this section, the Arsenin-Kunugui theorem is applied to treat some problems discussed earlier.

TheOREM 1.1. Let $B$ be a Borel subset of $X \times Y$ such that for each $x, B_{x}$ is a $K_{\sigma}$ set (the union of countably many compact sets.) Then for each $\alpha<\omega_{1}, D_{Y}^{(\alpha)}(B)$ is a Borel set and each $X$-section of $D_{Y}^{(\alpha)}(B)$ is a $K_{\sigma}$ set.

Proof. Let $\left\{U_{n}\right\}_{n=1}^{\infty}$ be a countable base for the topology of $Y$. For each $n$, let $W_{n}=\left\{x:\left[B \cap\left(X \times U_{n}\right)\right]_{x}\right.$ consists of a single point $\}$. According to a theorem of Braun [1], $W_{n}$ is a Borel subset of $X$. Then

$$
D_{Y}^{(1)}(B)=B-\bigcup_{n=1}^{\infty}\left(W_{n} \times U_{n}\right),
$$

and $D_{Y}^{(1)}(B)$ is a Borel set.

Clearly, each $X$-section of $D_{Y}^{(1)}(B)$ is the intersection of a closed set with the corresponding $X$-section of $B$. Thus, $D_{Y}^{(1)}(B)$ has the properties stated in the conclusion.

If $D_{Y}^{(\alpha)}(B)$ has the stated properties, then by the preceding argument $D_{Y}^{(\alpha+1)}(B)$ has the stated properties.

Now, suppose $\alpha$ is a countable limit ordinal. Let $\left\{\alpha_{n}\right\}_{n=1}^{\infty}$ be an increasing sequence of ordinals coverging to $\alpha$. Clearly, $D_{Y}^{(\alpha)}(B)=$ $\bigcap_{n=1}^{\infty} D_{Y}^{\left(\alpha_{n}\right)}(B)$. So, $D_{Y}^{(\alpha)}(B)$ is Borel. Let $x \in X$. Then $T=B_{x}$ is a $K_{\sigma}$ set. If $(x, y) \in T-\left(D_{Y}^{(\alpha)}(B)\right)_{x}$ then there is some $n$ such that $U_{n} \cap T$ misses $\left(D_{Y}^{(\alpha)}(B)\right)_{x}$. Thus, $T-\left(D_{Y}^{(\alpha)}(B)_{x}\right.$ is open with respect to $T$ and $\left(D_{Y}^{(\alpha)}(B)\right)_{x}$ is the intersection of a closed set with the $K_{\sigma}$ set T. Thus, $\left(D_{Y}^{(\alpha)}(B)\right)_{x}$ is a $K_{\sigma}$ set.

THEOREM 1.2. Let $E$ be a subset of $X \times Y$ which is universal 
for the family of countable, closed subsets of $Y$. If $Y$ is uncountable, then $E$ is not analytic.

Proof. Let us suppose that $E$ is analytic and $Y$ is uncountable. Let $P$ be a compact perfect subset of $Y$. Let $A=(X \times P) \cap E$. Then $A$ is an analytic subset of $X \times P$ which is universal for the countable closed subsets of $P$.

Let $F$ be a closed subset of $(X \times P) \times I$ such that for each pair $(x, p) \in X \times P, F_{(x, p)}$ is uncountable if and only if $(x, p) \in A[4, \mathrm{p}$. $497]$.

Let $\varphi$ be the map of $X$ into, $2^{P \times I}$, the space of closed subsets of $P \times I$ defined by $\varphi(x)=F_{x}$, where $F_{x}$ is regarded as a closed subset of $X \times(P \times I)$. $\quad 2^{P \times I}$ is considered to have the topology generated by the Hausdorff metric.

Now, it follows from the Arsenin-Kunugui theorem that $\varphi$ is a Borel mapping of $X$ into $2^{P \times I}$ [2, p. 379].

Let $M=\left[2^{P} \times 2^{P \times I} \times \varphi(X)\right] \cap G$, where $G=\left\{(D, F, B) \in 2^{P} \times\right.$ $2^{P \times I} \times 2^{P \times I}: D=\pi_{P}(F)$, and $F \subset B$ and if $V$ is open in $P$ and $F \cap$ $(V \times I) \neq \varnothing$, then $\exists x \varepsilon V$ such that $F_{x}$ is uncountable\}.

For the moment, let us assume that $M$ is analytic. This implies that $\pi_{1}(M)=\left\{D \in 2^{P}: D\right.$ is countable $\}$, is an analytic subset of $2^{P}$. But, this set is known not to be analytic in $2^{P}$, [5, p. 72].

Thus, the proof will be complete, once $M$ is shown to be analytic. In order to see this it suffices to show that the set $L$ of the next lemma is analytic.

LEMMA 1.3. Let $L=\left\{F \in 2^{P \times I}\right.$ : if $V$ is an open subset of $P$ and $V \cap \pi_{1}(F) \neq \varnothing$, then there is some $x \in V$ such that $F_{x}$ is uncountable\}. Then $L$ is an analytic subset of $2^{P \times T}$.

Proof. Let $\left\{V_{n}\right\}_{n=1}^{\infty}$ be a countable base for the topology of $P$. Let $L_{n}=\left\{F \in 2^{P \times I}\right.$ : either $V_{n} \cap \pi_{1}(F)=\varnothing$ or there is some $x \in V_{n}$ such that $F_{x}$ is uncountable\}. Evidently, $L=\bigcap_{n=1}^{\infty} L_{n}$.

Let $g$ be the map of $P \times 2^{I}$ into $2^{P \times I}$ defined by $g(x, T)=\{x\} \times T$. Let $H=\left\{T \in 2^{I}: T\right.$ is uncountable $\}$. Then $H$ is an analytic set [5, p. 72]. Clearly, $g$ is continuous and therefore $W=g(P \times H)$ is an analytic subset $2^{I \times I}$. Let $S=\left\{(K, F) \in 2^{P \times I} \times 2^{P \times I}: K \in W\right.$ and $\left.K \subset F\right\}$. Then $S$ is analytic in $2^{P \times I} \times 2^{P \times I}$ and

$$
L_{n}=\pi_{1}(S) \cup\left\{F \in 2^{P \times I}: V_{n} \cap \pi_{1}(F)=\varnothing\right\} .
$$

It follows from this expression that each $L_{n}$ is analytic and therefore $L$ is analytic. 
Let us note that by following the arguments of this section, we have:

TheOREM 1.4. Let $Y$ be Polish and $\mathscr{C}$ a family of closed countable subsets of $Y$ such that each closed countable subset of $Y$ is a subset of some member of $\mathscr{C}$. If $Y$ is uncountable and $E$ is an universal set for $\mathscr{C}$ in $X \times Y$, then $E$ is not analytic.

Finally it was noted earlier that if $B$ is Borel in $X \times P$ and each $X$-section of $B$ is closed, then $\varphi: X \rightarrow 2^{P}$ defined by $\varphi(x)=B_{x}$ is a Borel map of $X$ into $2^{P}$. This leads to the following

EXAMPLE. There is an analytic subset $A$ of $I \times I$ such that each vertical section of $A$ is closed and yet $\left\{A_{x}: x \in I\right\}$ is not an analytic subset of $2^{I}$ of $2^{I}$ (regard each $A_{x}$ as a subset of $I$ ).

Before constructing such a set, let us note the following

Lemma. If $G$ is an analytic subset of $2^{I}$, then $T=\{x: x \in H$, for some $H \in G\}$ is an analytic subset of $I$.

Proof. Let $M=\left\{(t, H): t \in H\right.$ and $\left.H \in 2^{I}\right\}$. Then $M$ is a closed subset of $I \times 2^{I}$ and $T=\pi_{1}(M \cap(I \times G))$.

Construction. Let $E$ be an analytic nonborel subset of $I$ and let $A=\{(x, y): x=y$ or $(x \in E$ and $y=0)\}$. Then $A$ is an analytic subset of $I \times I$.

Suppose $J=\left\{A_{x}: x \in I\right\}$ is analytic in $2^{I}$. Let $S=\{\{x\}: x \in I\}$. Then $S$ is a closed subset of $2^{I}$ and $W=S \cap J$ is an analytic subset of $2^{I}$. Therefore,

$$
T=\{t: t \in H, \text { for some } H \in W\}
$$

is an analytic subset of $I$. But, $T=I-E$. This is a contradiction.

2. Theorem C. In this section Theorem $\mathrm{C}$ is proven. The proof follows to a great extent the outline given by Lusin in [7, p. 247].

Let $A$ be an analytic subset of $X \times Y$ such that each $X$-section of $A$ is countable. The proof is by contradiction. Thus, if $B$ is a Borel set containing $A$, then some $X$-section of $B$ is uncountable.

First, let us realize $A$ as the projection of a $G_{o}$ set. Let $\left\{U_{n}\right\}_{n=0}^{\infty}$ be a decreasing sequence of open subsets of $(X \times Y) \times I$, where $I$ is the unit interval such that

where

$$
\pi_{X Y}(G)=A,
$$




$$
G=\bigcap_{n=0}^{\infty} U_{n}
$$

Lemma 2.1. There is an open parallelepiped $R$ in $X \times Y \times I$ such that

(i) diameter $(R)<1$.

(ii) $\bar{R} \subset U_{0}$.

(iii) if $B$ is a Borel subset of $X \times Y$ containing $\pi_{X Y}(R \cap G)$, then there are uncountably many $x$ 's such that $B_{x}$ is uncountable.

Proof. Let $\mathscr{B}$ be a countable base for the topology of $U_{0}$ consisting of open parallelepipeds, $B$, such that $\operatorname{diam}(B)<1$ and $\bar{B} \subset U_{0}$. Let $\left\{T_{n}\right\}_{n=1}^{\infty}$ be a sequence consisting of all the members of $\mathscr{B}$ which have nonempty intersection with $G$.

Let us suppose that for each $n$, there are a Borel set $B_{n}$ containing $\pi_{X Y}\left(T_{n} \cap G\right)$ and a countable subset $C_{n}$ of $X$ such that if $x \notin C_{n}$, then $B_{n x}$ is countable.

Let $B=\left(\bigcup_{n=1}^{\infty} B_{n}-\bigcup_{n=1}^{\infty}\left(C_{n} \times Y\right)\right) \cup\left(A \cap \bigcup_{n=1}^{\infty}\left(C_{n} \times Y\right)\right)$.

Clearly, $B$ is a Borel set containing $A$ and every $X$-section of $B$ is countable. This contradiction establishes Lemma 2.1.

Let $L$ be a sieve which sifts $\pi_{X Y}(R \cap G)(R \cap G)$ [5, 7]. Thus, $L$ is a map from the rationals into $2^{X \times Y}$ such that $a \in A$ if and only if $I_{L}(a)$ is not well-ordered, where $I_{L}(a)=\{q: a \in L(q)\}$. Also, for each countable ordinal $\alpha$, let $C_{\alpha}(L)$ be the $\alpha$ th constituent of $L: C_{\alpha}(L)=\left\{a\right.$ : the order type of $I_{L}(\alpha)$ is $\left.\alpha\right\}$. Reinterpreting Lemma 2.1, we have

Lemma 2.2. For every $\alpha<\omega_{1}$, there is some $x \in X$ (in fact uncountably many) such that $\left(\mathbf{U}_{\alpha<\beta} C_{\beta}(L)\right)_{x}$ is uncountable.

Proof. Let us assume the contrary. Then there is an $\alpha_{0}<\omega_{1}$ such that for every $x,\left(\mathbf{U}_{\alpha_{0}<\beta} C(L)\right)_{x}$ is countable.

But, then

$$
B=X \times Y-\bigcup_{\beta \leq \alpha_{0}} C_{\beta}(L)
$$

is a Borel set containing $\pi_{X Y}(R \cap G)$ and for every $x, B_{x}$ is countable.

Let $\left\{F_{n}\right\}_{n=1}^{\infty}$ be a sequence consisting of all basic open rectangles, $F$, lying in $\pi_{X Y}\left(R \cap U_{1}\right)$ such that there is a basic open subinterval, $H$, of $I$ such that $\overline{F \times H} \subset R \cap U_{1}$, diam $(F \times H)<1 / 2$ and $(F \times H) \cap G \neq \varnothing$.

For each $n$, let $K_{n}$ map $Q$ into the Borel subsets of $X \times Y$ by $K_{n}(q)=L(q) \cap F_{n}$. Thus, $K_{n}$ is a sieve which sifts $\pi_{X Y}(R \cap G) \cap F_{n}$. Moreover, the constituents are related as follows:

$$
C_{0}\left(K_{n}\right)=C_{0}(L) \cup\left((X \times Y)-F_{n}\right) \text { and } C_{\alpha}\left(K_{n}\right)=C_{\alpha}(L) \cap F_{n},
$$


for $\alpha>0$.

LeMmA 2.3. There are positive integers $n$ and $p$ such that $\bar{F}_{n} \cap \bar{F}_{p}=\varnothing$ and for every $\alpha<\omega_{1}$, there is some $x$ such that $\left(\mathbf{U}_{\alpha<\beta} C_{\beta}\left(K_{n}\right)\right)_{x}$ and $\left(\mathbf{U}_{\alpha<\beta} C_{\beta}\left(K_{p}\right)\right)_{x}$ are both uncountable.

Proof. For each $\alpha<\omega_{1}$, let $x(\alpha)$ be a point of $X$ such that $W_{\alpha}=\left(\mathbf{U}_{\alpha<\beta} C_{\beta}\left(L_{0}\right)\right)_{x(\alpha)}$ is uncountable. Let $n(\alpha)$ and $p(\alpha)$ be positive integers such that $\overline{F_{n(\alpha)}} \cap \overline{F_{p(\alpha)}}=\varnothing$ and both $F_{n(\alpha)}$ and $F_{p(\alpha)}$ meet $W_{\alpha}$ in an uncountable set. There exist $n$ and $p$ such that $n=n(\alpha)$ and $p=p(\alpha)$ for uncountably many $\alpha$ 's. Clearly, these integers have the required properties.

Let us reinterpret Lemma 2.3.

LEMmA 2.4. If $B_{1}$ and $B_{2}$ are disjoint Borel sets containing $D_{1}=F_{n} \cap \pi_{x y}(R \cap G)$ and $D_{2}=F_{p} \cap \pi_{x y}(R \cap G)$ respectively, then there are uncountably many $x$ 's such that both $B_{1 x}$ and $B_{2 x}$ are uncountable.

Proof. Let $B_{1}$ and $B_{2}$ be disjoint Borel sets containing $D_{1}$ and $D_{2}$ respectively. There is an ordinal $\alpha<\omega_{1}$ such that

$$
E_{\alpha}^{n}=\bigcup_{\alpha<\beta} C_{\beta}\left(K_{n}\right) \subset B_{1} \text { and } E_{\alpha}^{p}=\bigcup_{\alpha<\beta} C_{\beta}\left(K_{p}\right) \subset B_{2} .
$$

But, there are uncountably many $x$ 's such that $E_{\alpha x}^{n}$ and $E_{\alpha x}^{p}$ are uncountable.

Lemma 2.5. There is an open parallelepiped $R_{0}$ such that:

(i) diameter $\left(R_{0}\right)<1 / 2$.

(ii) $\overline{R_{0}} \subset R \cap U_{1}$.

(iii) $\pi_{x y}\left(R_{0}\right) \subset F_{n}$.

(iv) if $B_{1}$ and $B_{2}$ are disjoint Borel sets containing

$D_{0}=\pi_{x y}\left(R_{0} \cap G\right)$ and $D_{2}=F_{p} \cap \pi_{x y}(R \cap G)$ respectively, then there are uncountably many $x$ 's such that $B_{1 x}$ and $B_{2 x}$ are uncountable.

Proof. Let $\left\{S_{n}\right\}_{n=1}^{\infty}$ be a sequence of all basic open parallelepipeds satisfying (i), (ii), and (iii) which also have nonempty intersection with $G$.

Suppose that for each $n$, there exist disjoint Borel sets $M_{n}$ and $N_{n}$ containing $\pi_{x y}\left(S_{n} \cap G\right)$ and $D_{2}$ respectively such that $C_{n}=\left\{x: M_{n x}\right.$ and $N_{n x}$ are uncountable\} is countable.

Let $B_{1}=\bigcup_{n=1}^{\infty} M_{n}$ and $B_{2}=\bigcap_{n=1}^{\infty} N_{n}$. Then $B_{1}$ and $B_{2}$ are disjoint Borel sets containing $D_{1}$ and $D_{2}$ respectively. Let $x$ be such that $B_{1 x}$ and $B_{2 x x}$ are uncountable and yet $x \notin \bigcap_{n=1}^{\infty} C_{n}$. Then each $M_{n x}$ is countable. This contradiction establishes Lemma 2.5. 
By a similar argument one can show that there is an open parallelepiped $R_{1}$ such that

(i) diameter $\left(R_{01}\right)<1 / 2$.

(ii) $\overline{R_{1}} \subset R \cap U_{1 .}$.

(iii) $\pi_{x y}\left(R_{1}\right) \subset F_{p}$.

(iv) if $B_{1}$ and $B_{2}$ are disjoint Borel sets containing $D_{0}$ and $D_{1}=\pi_{x y}\left(R_{1} \cap G\right)$ respectively, then there are uncountably many $x$ 's such that $B_{1 x}$ and $B_{2 x}$ are uncountable.

Finally, it may be shown by induction:

TheOREM 2.6. For each finite sequence $\left(e_{1}, \cdots, e_{n}\right)$ of zeros and ones there is an open parallelepiped $R_{e_{1} \cdots e_{n}}$ of $(X \times Y) \times I$ such that for each $n$ :

(i) all $R_{e_{1} \cdots e_{n^{e}+1}}$ have diameter $<1 / 2^{n}$,

(ii) $\overline{R_{e_{1} \cdots e_{n} 0}} \cup \overline{R_{e_{1} \cdots e_{n}}} \subset R_{e_{1} \cdots e_{n}} \cap u_{n}$,

(iii) $\overline{R_{e_{1} \cdots e_{n} 0}} \cap \overline{R_{e_{1} \cdots e_{n^{1}}}}=\varnothing$,

(iv) $\pi_{x y}\left(R_{e_{1} \cdots e_{n} 0}\right) \cap \pi_{x y}\left(R_{e_{1} \cdots e_{n^{1}}}\right)=\varnothing$,

(v) if $B_{e_{1} \cdots e_{n} e_{n+1}}$, where $\left(e_{1}, \cdots, e_{n+1}\right) \in\{0,1\}^{n+1}$, are disjoint Borel sets containing $\pi_{x y}\left(R_{e_{1} \cdots e_{n} e_{n+1}} \cap G\right)$, then there are uncountably many $x$ 's such that all the sets $\left(B_{e_{1} \cdots e_{n+1}}\right)_{x}$ are uncountable. For each finite sequence $\left(e_{1} \cdots e_{n}\right)$ of 0 's and 1 's, let

$$
P\left(e_{1} \cdots e_{n}\right)=\left(x\left(e_{1} \cdots e_{n}\right), y\left(e_{1} \cdots e_{n}\right), t\left(e_{1} \cdots e_{n}\right)\right)
$$

be a point of $R_{e_{1} \cdots e_{n}}$.

It can be checked that for each infinite sequence $e=\left\{e_{k}\right\}_{k=1}^{\infty}$ from $2^{\prime \prime}$ the sequence $\{P(e \mid n)\}_{n=1}^{\infty}$ converges to some point $P(e)=(x(e), y(e), t(e))$ of $G$. It follows from Theorem 2.6, that if $e \neq e^{\prime}$ then $y(e) \neq y\left(e^{\prime}\right)$.

It is now shown that if $e \neq e^{\prime}$, then $x(e)=x\left(e^{\prime}\right)$ :

It follows from the properties listed in Theorem 2.6 that for each $n$, there is some $x_{n}$ of $X$ and points $y_{n}$ and $y_{n}^{\prime}$ of $Y$ such that $\left(x_{n}, y_{n}\right) \in \pi_{x y}\left(R_{e \mid n}\right)$ and $\left(x_{n}, y_{n}^{\prime}\right) \in \pi_{x y}\left(R_{e^{\prime} \mid n}\right)$.

Therefore $x_{n} \rightarrow x(e)$ and $x_{n} \rightarrow x\left(e^{\prime}\right)$. Thus, $x(e)=x\left(e^{\prime}\right)$.

This last result implies that $A_{x(e)}$ is uncountable. This contradiction establishes Theorem $\mathrm{C}$.

3. A Proof of Theorem L. In this section, Theorem L, as stated in the introduction, is proven. As was noted in the introduction, it suffices to consider the following situation: $E$ is an analytic subset of $X \times Y$ such that each section of $E$ is scattered and for every $\alpha, \alpha<\omega_{1}, D_{Y}^{(\alpha)}(E) \neq \varnothing$.

The aim of this section is to prove Theorem $L$ by showing that these assumptions concerning $E$ lead to some $X$-section of $E$ con- 
taining a dense-in-itself set. From this contradiction, it follows that Theorem L holds.

To begin with, we have from Theorem C that $E=\bigcup_{n=1}^{\infty} \Gamma_{n}$, where for each $n, \Gamma_{n}$ is an analytic set which is uniform with respect to $X$. (Recall that $\Gamma_{n}$ is uniform means each $\left(\Gamma_{n}\right)_{x}$ has cardinality less than 2.)

All rings and bands considered in this section are with respect to $E$.

Let $n$ be such that $\Gamma=\Gamma_{n}$ has the property that for every countable ordinal $\alpha, \Gamma \cap D_{Y}^{(\alpha)}(E) \neq \varnothing$. The existence of such a $\Gamma_{n}$ follows from a simple cardinality argument.

Lemma 3.1. For each $\varepsilon>0$, there is a positive number $\varepsilon^{\prime}, \varepsilon^{\prime}<\varepsilon$ such that $\Gamma$ and $\mathscr{R}_{\varepsilon^{\prime}}^{\S}(\Gamma)$ are simultaneously unbounded.

Proof. Let $\varepsilon>0$. For each $\alpha, \alpha<\omega_{1}$, let $\left(x_{\alpha}, y_{\alpha}\right) \in \Gamma \cap D_{y}^{(\alpha+1)}(E)$ and let $\varepsilon(\alpha)$ be a positive rational such that $\left(\mathscr{R}_{\varepsilon(\alpha)}^{\varepsilon}(\Gamma)\right)_{x(\alpha)} \cap D_{Y}^{(\alpha)}(E) \neq \varnothing$. There must be some $\varepsilon^{\prime}$ which is $\varepsilon(\alpha)$ for uncountably many $\alpha$ 's. Clearly, $\Gamma$ and $\mathscr{R}_{\varepsilon^{\prime}}^{\varepsilon}(\Gamma)$ are simultaneously unbounded.

Now, let $\varepsilon^{\prime}$ be such that $\Gamma$ and $\mathscr{R}_{\varepsilon^{\prime}}^{1}(\Gamma)$ are simultaneously unbounded.

According to Theorem $\mathrm{C}$,

$$
\mathscr{R}_{\varepsilon^{\prime}}^{1}(\Gamma)=\bigcup_{n=1}^{\infty} T_{n},
$$

where each $T_{n}$ is an analytic set which is uniform with respect to $X$.

Lemma 3.2. There exists some $k$ such that $\Gamma$ and $T=T_{k}$ are simultaneously unbounded.

This lemma also follows by a simple cardinality argument.

The set of all irrationals will be denoted by $J$. If $k_{1} \cdots k_{p}$ is a finite sequence of positive integers, $\mathscr{N}_{k_{1} \cdots k_{p}}$ denotes the set of irrationals with $k_{1} \cdots k_{p}$ as the initial sequence in its continued fraction expansion.

Let $\varphi_{0}$ be a continuous map of $J$ onto $\Gamma$; $\varphi_{1}$ a continuous map of $J$ onto $T$, and $\psi$ a continuous map of $J$ onto $\pi_{X}(E)$.

Let $\delta=\min \left(\varepsilon^{\prime} / 2,1 / 2\right)$. Then the bands $B_{\delta}\left(\varphi_{0}(J)\right)$ and $B_{\delta}\left(\varphi_{1}(J)\right)$ are disjoint.

LEMMA 3.3. There is a positive rational $\delta^{\prime}, \delta^{\prime}<\delta$, such that the sets $\varphi_{0}(J), \varphi_{1}(J), \mathscr{R}_{\dot{o}^{\prime}}^{\delta}\left(\varphi_{0}(J)\right)$ and $\mathscr{R}_{\delta^{\prime}}^{\delta}\left(\varphi_{1}(J)\right)$ are simultaneously unbounded on $\psi(J)$. 
Proof. For each $\alpha, \alpha<\omega_{1}$, let $x(\alpha)$ be a point of $X$ such that $\left(\varphi_{i}(J)\right)_{x(\alpha)} \cap D_{Y}^{(\alpha+1)}(E) \neq \phi, i=0,1$. For each countable ordinal $\alpha$, let $\delta(\alpha)$ be a rational, $0<\delta(\alpha)<\delta$ such that the intersection of $D_{Y}^{(\alpha)}(E)$ with each of $\left(\varphi_{0}(J)\right)_{x},\left(\varphi_{1}(J)\right)_{x},\left(\mathscr{R}_{\delta(\alpha)}^{\delta}\left(\varphi_{0}(J)\right)\right)_{x}$ and $\left(\mathscr{R}_{\delta(\alpha)}^{\delta}\left(\varphi_{1}(J)\right)\right)_{x}$ is nonempty. There must be some $\delta^{\prime}$ which is $\delta(\alpha)$ for uncountably many $\alpha$ 's. Clearly, $\delta^{\prime}$ satisfies the requirements of the lemma.

Since the two rings of the preceding lemma, may be expressed as the union of countably many analytic sets which are uniform with respect to $X$, we have:

LEMMA 3.4. There exist continuous functions $\varphi_{11}$ and $\varphi_{12}$ of $J$ into $E$ such that $\varphi_{11}(J) \subset \mathscr{R}_{\delta^{\prime}}^{\delta}\left(\varphi_{0}(J)\right), \varphi_{12} \subset \mathscr{R}_{\delta^{\prime}}^{\delta}\left(\varphi_{1}(J)\right)$ and $\varphi_{0}(J), \varphi_{1}(J)$, $\varphi_{11}(J)$, and $\varphi_{12}(J)$ are simultaneously unbounded on $X$.

Since $\pi_{x}(E)=\bigcup_{t=1}^{\infty} \psi\left(\mathscr{N}_{t}\right)$, there must be some $t_{1}$ such that $\varphi_{0}(J)$, $\varphi_{1}(J), \varphi_{11}(J)$, and $\varphi_{12}(J)$ are simultaneously unbounded on $\psi\left(\mathscr{N}_{t_{1}}\right)$. There must be an integer $s_{1}^{0}$ such that $\varphi_{0}\left(\mathscr{N}_{s_{1}^{0}}\right), \varphi_{1}(J), \varphi_{11}(J)$ and $\varphi_{12}(J)$ are simultaneously unbounded on $\psi\left(\mathscr{N}_{t_{1}}\right)$. Continuing in this manner, we have:

LEMMA 3.5. There exist integers $t_{1}, s_{1}^{0}, s_{1}^{1}, s_{1}^{11}, s_{2}^{12}$ such that $\varphi_{0}\left(\mathscr{N}_{s_{1}^{0}}^{0}\right), \varphi_{1}\left(\mathscr{N}_{s_{1}^{1}}^{1}\right), \varphi_{11}\left(\mathscr{N}_{\mathbf{s}_{1}^{11}}^{11}\right)$, and $\varphi_{12}\left(\mathscr{N}_{s_{1}^{12}}^{12}\right)$ are simultaneously unbounded on $\psi\left(\mathscr{N}_{t_{1}}\right)$. Also, there is a positive number $\varepsilon_{1}$ such that the $\varepsilon_{1}-$ bands of the first four sets are disjoint.

This completes the first stage of an induction process. The second stage is completed by the following procedure. First, let $\delta=\min \left(\varepsilon_{1}, 1 / 4\right)$. Second, obtain a $\delta^{\prime}, 0<\delta^{\prime}<\delta$ such that the eight sets: $\varphi_{0}\left(\mathscr{N}_{s_{1}^{0}}^{0}\right), \varphi_{1}\left(\mathscr{N}_{s_{1}^{1}}^{1}\right), \varphi_{11}\left(\mathscr{N}_{s_{1}^{11}}^{11}\right), \varphi_{12}\left(\mathscr{N}_{s_{1}^{12}}^{12}\right), \mathscr{R}_{\delta^{\prime}}^{\delta}\left(\varphi_{0}\left(\mathscr{N}_{s_{1}^{0}}\right)\right), \mathscr{R}_{\delta^{\prime}}^{o}\left(\varphi_{1}\left(\mathscr{N}_{s_{1}^{1}}\right)\right)$, $\mathscr{R}_{\delta^{\prime}}^{\delta}\left(\varphi_{11}\left(\mathscr{N}_{s_{1}^{1}}^{1)}\right)\right.$ and $\mathscr{R}_{\dot{o}^{\prime}}^{\delta}\left(\varphi_{12}\left(\mathscr{N}_{\boldsymbol{s}_{1}^{1}}^{1}\right)\right)$ are simultaneously unbounded on $\psi\left(\mathscr{N}_{t_{1}}\right)$. Third, obtain continuous functions $\varphi_{21}, \varphi_{22}, \varphi_{23}, \varphi_{24}$ of $J$ into the respective rings such that the first four sets of the preceding sentence and $\varphi_{2 i}(J), i=1,2,3,4$, are simultaneously unbounded on $\psi\left(\mathscr{N}_{t_{1} t_{2}}\right)$. Fourth, fix $t_{2}$ so that these eight graphs are unbounded on $\Psi\left(\mathscr{N}_{t_{1} t_{2}}\right)$. Finally obtain integers: $s_{1}^{0}, s_{2}^{1}, s_{2}^{11}, s_{2}^{12}$ and integer pairs: $\left(s_{1}^{21}, s_{2}^{21}\right),\left(s_{1}^{22}, s_{2}^{22}\right),\left(s_{1}^{23}, s_{2}^{23}\right),\left(s_{1}^{24}, s_{2}^{24}\right)$ such that the sets: $\varphi_{0}\left(\mathscr{N}_{s_{1} s_{2} s_{2}}\right) \varphi_{1}\left(\mathscr{N}_{s_{1}^{1} s_{2}^{1}}\right)$ and $\varphi_{i j}\left(\mathscr{N}_{s_{12}^{i j}}^{i}\right), i=1,2, j \leqq 2^{i}$ are simultaneously unbounded on $\psi\left(\mathscr{N}_{t_{1} t_{2}}\right)$.

Continuing this process by induction, we have:

THEOREM 3.6. For each $n \in N$ and $p \leqq 2^{n}$, there is a continuous map $\varphi_{n p}$ of $J$ into $A$, a point $\sigma^{n p}=\left(s_{1}^{n p}, s_{2}^{n p}, \cdots\right)$ of $J$ and points $\sigma^{0}=\left(s_{1}^{0}, s_{2}^{0}, \cdots\right), \sigma^{1}=\left(s_{1}^{1}, s_{2}^{1}, \cdots\right)$, and $\tau=\left(t_{1}, t_{2}, t_{3}, \cdots\right)$ of $J$ such that 
( i ) the sets $\varphi_{n p}(J)$ are disjoint and uniform with respect to $X$

(ii) if $n \in N$, then the sets $\varphi_{k p}\left(\mathscr{N}_{\left.\sigma^{k p}\right|_{n}}\right), k \leqq n, p \leqq 2^{k}$, and $\varphi_{0}\left(\mathscr{N}_{\sigma^{0} \mid n}\right)$ and $\varphi_{1}\left(\mathscr{N}_{\sigma^{1} \mid n}\right)$ are simultaneously unbounded on $\psi\left(\mathscr{N}_{\tau \mid n}\right)$,

(iii) if $1 \leqq k<l$ and $p \leqq 2^{k}$, then there is some $j \leqq 2^{l}$ such that $\varphi_{l t}(J)$ lies in the $2^{-l}$-band of $\varphi_{k p}\left(\mathscr{N}_{\sigma^{k p} p_{n}}\right)$.

We now proceed with the

Proof of Theorem L. Let $x=\psi(\tau)$ and $\left(x_{k p}, y_{k p}\right)=\varphi_{k p}\left(\sigma^{k p}\right), k \in N$, $p \leqq 2^{k}$. Thus, $\left(x_{k p}, y_{k p}\right) \in E$, for $k \in N$ and $p \leqq 2^{k}$.

It is now shown that $x_{k p}=x$. For each $i>k, \varphi_{k p}\left(\mathscr{N}_{\sigma^{k p} \mid i}\right)$ is unbounded on $\psi\left(\mathscr{N}_{\tau \mid i}\right)$. Let $z_{i} \in \mathscr{N}_{\boldsymbol{o}^{k p \mid i}}$ with $\varphi_{k p}\left(\boldsymbol{z}_{i}\right)=\left(x_{i}, \boldsymbol{y}_{i}\right)$ and $x_{i} \in \psi\left(\mathscr{N}_{\tau \mid i}\right)$. As $i \rightarrow \infty, z_{i} \rightarrow \sigma^{k p}$ and therefore $x_{i} \rightarrow x_{k p}$. But $x_{i} \rightarrow x$. So, $x=x_{k p}$.

Thus, $E_{x}$ contains $D=\left\{\left(x_{k p}, y_{k p}\right): k \in N\right.$ and $\left.p \leqq 2^{k}\right\}$.

It is now shown that $D$ is dense-in-itself. To see this consider a point $\left(x, y_{k p}\right)$ of $D$ and a positive number $\varepsilon$. Pick $n>k$ so that $2^{-n}<\varepsilon$. By property (iii) of Theorem 3.6, there is some $j \leqq 2^{n}$ such that $\varphi_{n j}(J)$ lies in the $1 / 2 n$-band of $\varphi_{k p}\left(\mathscr{N}_{\left.\sigma^{k p}\right|_{n}}\right)$. Thus, $\varphi_{n j}\left(\sigma^{n j}\right)$ and $\varphi_{k p}\left(\sigma^{k p}\right)$ both lie in this band. Since the points are different and have the same $X$-coordinate, $y_{n t}$ lies in the $\varepsilon$-neighborhood of $y_{k p}$ and $y_{n t} \neq y_{k p}$.

But, by the hypothesis of Theorem L, $E_{x}$ is scattered. This contradiction implies that the conclusion of Theorem $\mathrm{L}$ holds.

\section{REFERENCES}

1. S. Braun, Quelques theoremes sur les cribles boreliens, Fund Math., 20 (1933), 168-172

2. R. R. Kallman, Certain quotient spaces are countably separated, Illinois J. Math., 19 (1975), 378-388.

3. A. S. Kechris and D. A. Martin, A note on universal sets for classes of countable $G_{o}$ 's, Mathematika, 22 (1975), 43-45.

4. K. Kuratowski, Topology, vol. 1, Academic Press, New York and London, 1966.

5. — Topology, vol. 2, Academic Press, New York and London, 1968.

6. D. G. Larman and C. A. Rogers, The descriptive character of certain universal sets, Proc. London Math. Soc. (3), 27 (1973), 385-401.

7. N. Lusin, Lecons sur les Ensembles Analytiques et leurs Applications, Second (corrected) edition, Chelsea Publishing Co., New York, 1972.

Received March 15, 1976.

UNIVERSITY OF FLORIDA

GAINESVILLE, FL 32611 



\section{PACIFIC JOURNAL OF MATHEMATICS}

\section{EDITORS}

RICHARD ARENS (Managing Editor)

University of California

Los Angeles, California 90024

C. W. Curtis

University of Oregon

Eugene, OR 97403

C. C. MOORE

University of California

Berkeley, CA 94720

\section{J. DUGUNDJI}

Department of Mathematics University of Southern California Los Angeles, California 90007

R. Finn AND J. Milgram Stanford University Stanford, California 94305

ASSOCIATE EDITORS
E. F. BECKENBACH

B. H. NeUMANN

F. WOLF

K. YoshidA

\section{SUPPORTING INSTITUTIONS}

UNIVERSITY OF BRITISH COLUMBIA UNIVERSITY OF SOUTHERN CALIFORNIA CALIFORNIA INSTITUTE OF TECHNOLOGY STANFORD UNIVERSITY UNIVERSITY OF CALIFORNIA MONTANA STATE UNIVERSITY UNIVERSITY OF TOKYO UNIVERSITY OF NEVADA, RENO UNIVERSITY OF UTAH NEW MEXICO STATE UNIVERSITY OREGON STATE UNIVERSITY UNIVERSITY OF OREGON

WASHINGTON STATE UNIVERSITY UNIVERSITY OF WASHINGTON OSAKA UNIVERSITY 


\section{Pacific Journal of Mathematics}

\section{Vol. 74, No. $1 \quad$ May, 1978}

Gerald Arthur Anderson, Computation of the surgery obstruction groups

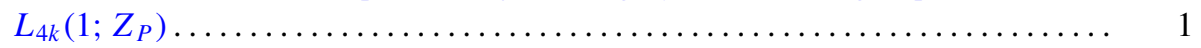

R. K. Beatson, The degree of monotone approximation ................ 5

Sterling K. Berberian, The character space of the algebra of regulated functions . . . 15

Douglas Michael Campbell and Jack Wayne Lamoreaux, Continua in the plane with

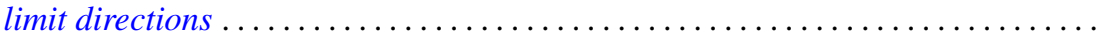

R. J. Duffin, Algorithms for localizing roots of a polynomial and the Pisot

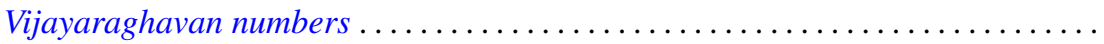

Alessandro Figà-Talamanca and Massimo A. Picardello, Functions that operate on

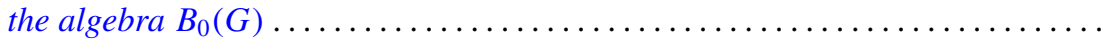

John Erik Fornaess, Biholomorphic mappings between weakly pseudoconvex

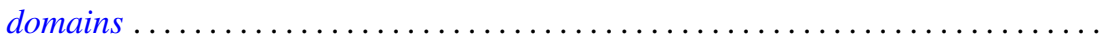

Andrzej Granas, Ronald Bernard Guenther and John Walter Lee, On a theorem of S.

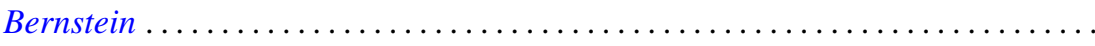

Jerry Grossman, On groups with specified lower central series quotients . .........

William H. Julian, Ray Mines, III and Fred Richman, Algebraic numbers, a constructive development . . . . . . . . . . . . . . . . . . . . . . .

Surjit Singh Khurana, A note on Radon-Nikodým theorem for finitely additive

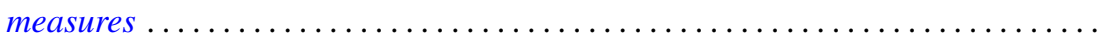

Garo K. Kiremidjian, A Nash-Moser-type implicit function theorem and nonlinear

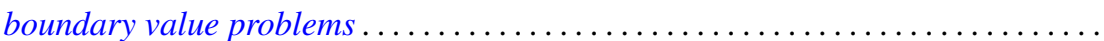

Ronald Jacob Leach, Coefficient estimates for certain multivalent functions ....

John Alan MacBain, Local and global bifurcation from normal eigenvalues. II . . 133

James A. MacDougall and Lowell G. Sweet, Three dimensional homogeneous algebras...

John Rowlay Martin, Fixed point sets of Peano continua ......

R. Daniel Mauldin, The boundedness of the Cantor-Bendixson order of some analytic sets...

Richard C. Metzler, Uniqueness of extensions of positive linear functions ..

Rodney V. Nillsen, Moment sequences obtained from restricted powers . .

Keiji Nishioka, Transcendental constants over the coefficient fields in differential elliptic function fields...

Gabriel Michael Miller Obi, An algebraic closed graph theorem

Richard Cranston Randell, Quotients of complete intersections by $\mathbf{C}^{*}$ actions . . 221

Bruce Reznick, Banach spaces which satisfy linear identities . .

Bennett Setzer, Elliptic curves over complex quadratic fields...

Arne Stray, A scheme for approximating bounded analytic functions on certain subsets of the unit disc.

Nicholas Th. Varopoulos, A remark on functions of bounded mean oscillation and bounded harmonic functions. Addendum to: "BMO functions and the

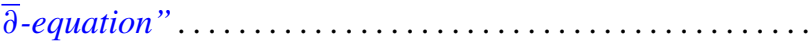

Charles Irvin Vinsonhaler, Torsion free abelian groups quasi-projective over their

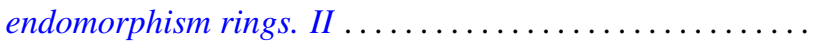

Thomas R. Wolf, Characters of $p^{\prime}$-degree in solvable groups ... 